\title{
Multidimensional Electron Diffraction-Microscopy of Cabotegravir Nanocrystals
}

\author{
Duncan N. Johnstone ${ }^{1}$, Royston C. B. Copley ${ }^{2}$, Rachel G. Graves ${ }^{3}$, Jeffrey Brum³ ${ }^{3}$ and Paul A. Midgley ${ }^{1}$ \\ 1. Department of Materials Science \& Metallurgy, University of Cambridge, Cambridge, UK. \\ 2. Physical Properties, CMC Analytical, GlaxoSmithKline Pharmaceuticals, Stevenage, UK. \\ 3. Physical Properties, CMC Analytical, GlaxoSmithKline Pharmaceuticals, Collegeville, USA.
}

Low-solubility active pharmaceutical ingredients (APIs) are increasingly formulated as nanosuspensions comprising pure API nanocrystals (i.e. $\mathrm{d}_{50} \sim 100 \mathrm{~nm}$ ) stabilized by surfactants or polymeric steric stabilizers [1]. The physicochemical properties of such nanosuspensions are characterized extensively using a range of predominantly bulk analytical techniques including imaging, spectroscopy and thermal analysis [1]. These techniques elucidate the overall properties of the nanosuspension but do not directly assess the local atomic structure of individual API nanocrystals. Revealing the local structure of individual nanocrystals could enhance product development, motivating technique development. Transmission electron microscopy (TEM) is a natural choice for nanoscale characterization but its application to APIs has been limited by electron beam damage. Here, we report on the development and application of new electron diffraction microscopy techniques, namely scanning electron diffraction (SED) microscopy [2] and continuous rotation tomography $[3,4]$, that minimize electron dose to overcome previous limitations. These techniques enable detailed characterization of individual cabotegravir (GSK1265744) nanocrystals, similar to those used in the formulation of cabotegravir long-acting nanosuspensions.

Cabotegravir is a potent integrase strand transfer inhibitor developed for HIV treatment that may be formulated as a long-acting injectable nanosuspension [5] comprising the low solubility nanocrystalline drug, with an average size of $\sim 200 \mathrm{~nm}$, in aqueous suspension and combined with surfactant system and mannitol as a tonicity agent. The nanosuspension is produced by wet bead nanomilling [5] of the crystalline drug followed by sterilization by gamma irradiation. For the purposes of this study, cabotegravir nanocrystals provide an industrially relevant example system for technique development. Samples were prepared using as milled nanopowder by deposition directly onto standard carbon coated TEM support grids. Continuous rotation tomography was performed under cryogenic conditions using a Thermo Fisher Titan Krios G3i operated at $300 \mathrm{kV}$ and SED microscopy was performed at room temperature and at $200 \mathrm{kV}$ using a Phillips Tecnai F20 retrofitted with a Nanomegas ASTAR system.

Crystallography and morphology of individual cabotegravir nanocrystals was investigated in three dimensions using continuous rotation electron diffraction tomography (EDT) [3] and bright-field (BF) TEM tomography [4]. A diffraction pattern or BF-TEM image was recorded for each tilt increment over a range of $\pm 60^{\circ}$ at a continuous tilt rate of $0.5^{\circ} \mathrm{s}^{-1}$ and exposure time of $1 \mathrm{~s}$ per frame, leading to an acquisition time of $\sim 3$ mins per tilt series. The BF tomography data were aligned by cross-correlation and tomographic reconstructions of the particles, as shown in Figure 1a, were achieved using the simultaneous iterative reconstruction technique with 20 iterations. The EDT data were processed to measure the unit cell parameters (orthorhombic, $a=7.26 \AA, b=7.29 \AA, c=32.3 \AA$ ) and reconstruct the diffraction data in three dimensions, as shown in Figure 1b. This combined real and reciprocal space tomography enables both the morphology and the crystallography of a particle to be assessed in parallel indicating, for example, that the larger faces of the particle are approximately perpendicular to the long axis of the unit cell, which is consistent with a simple Bravais-Friedel-Donnay-Harker interpretation, i.e. that the morphological importance is highest for facets associated with atomic planes with the largest interplanar spacing. Surface steps are also evident. 
Sub-nanocrystal structural variations may be assessed by SED microscopy, in which a two-dimensional electron diffraction pattern is recorded at every probe position as a nanometre sized electron beam is scanned across the specimen in a two-dimensional scan pattern. The average diffraction pattern from across one such nanocrystal, shown in Figure 2a, suggests it may be a single crystal. However, virtual dark-field images, formed by plotting the intensity within a selected region of the diffraction pattern as a function of probe position, suggest that there is a sharp change in orientation between the left and right sides of the crystal, which shows that there may be structural heterogeneity on the sub-nanocrystal level. This work demonstrates that newly developed cryo-electron diffraction microscopy techniques offer a whole new level of pharmaceutical nanocrystal characterization in multiple dimensions [6].

\section{References:}

[1] L Peltonen, Advanced Drug Delivery Reviews, 131 (2018), p. 101-115.

[2] D N Johnstone \& P A Midgley Microsc. Microanal., 23 (2017) p. 1192-1193.

[3] B L Nannenga et al, Nature Methods, 11 (2014), p. 927-930.

[4] V Migunov et al, Scientific Reports, 5 (2015), p. 14516.

[5] C Trezza et al, Current Opinion in HIV and AIDS, 10 (2015), p. 239-245.

[6] The authors acknowledge financial support from the GSK-Cambridge Varsity Alliance.

a

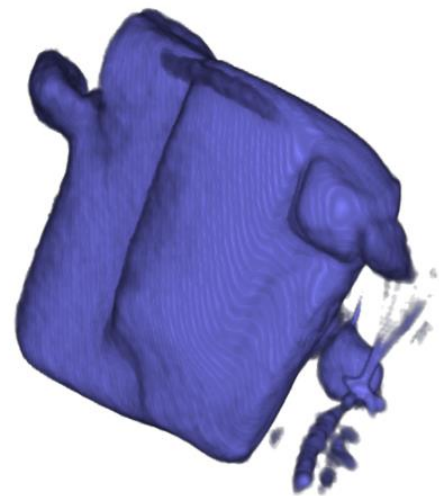

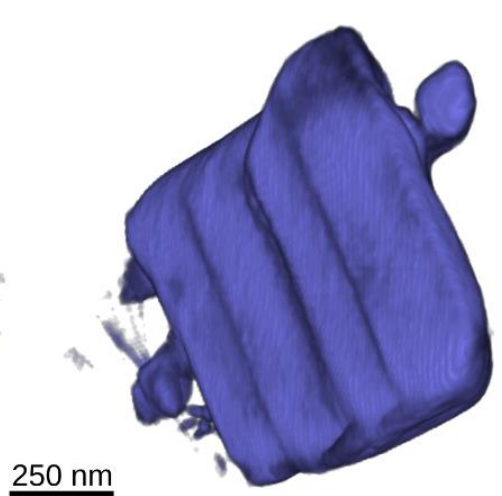

b

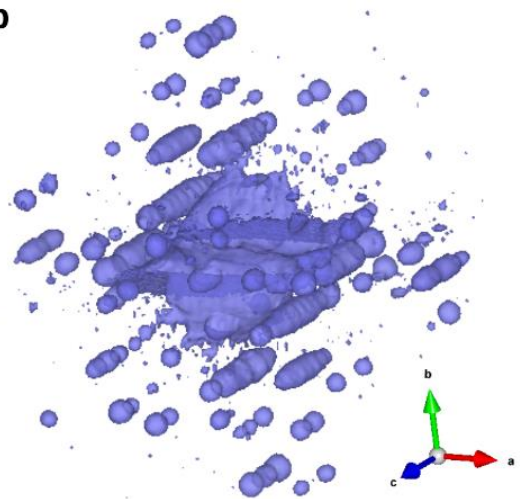

Figure 1. Three-dimensional reconstruction of cabotegravir nanocrystal in (a) real space, viewed in two orientations to show surface steps, and (b) reciprocal space using continuous rotation bright-field TEM tomography and continuous rotation electron diffraction tomography respectively.
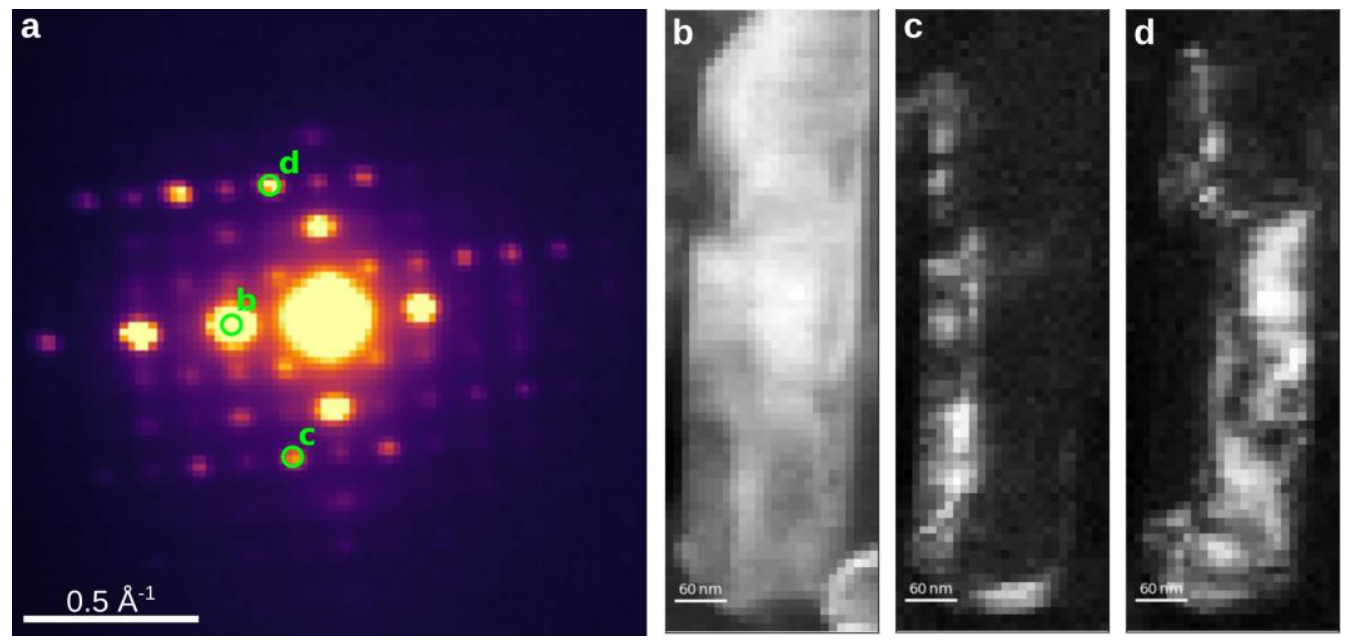

Figure 2. SED microscopy of a cabotegravir nanoparticle. (a) Average diffraction pattern from particle. (b-d) Virtual dark-field images associated with integration windows marked in (a). 